\title{
FIRST CAUSES: DIVINE AND HUMAN
}

\section{UWE MEIXNER}

University of Augsburg

\begin{abstract}
The paper analyzes the concept of a first cause, both for event causation and for agent causation. It turns out that one is rather ready to believe in the existence of first causes that are events, but not in the existence of first causes that are agents. The paper, however, develops and defends a complex argument to the conclusion that there is a first agent-cause. One version of that argument proves - not necessarily the existence of God - but still the existence of a godlike agent. Finally, the notion of a first agent-cause is employed for an analysis of freely willed human action.
\end{abstract}

There is a kind of causation where the cause is sufficient for the realization of the effect (that is, for the realization of what is caused), the effect being some event: an entity involving a finite spatiotemporal region, particulars, and properties (relational and non-relational ones) had by these particulars within that spatiotemporal region:

(A1) Effects (i.e., what is caused) are always events.

In sufficient causation, the cause determines the coming-about of the effect-event; the cause does not make the effect-event merely probable, or more probable than it would be without the cause, and the cause is not merely an indispensable factor for the coming-about of the effect-event. In sufficient event-causation, the coming-about of an event determines the coming-about of the effect-event; in sufficient agent-causation, simply the agent determines the coming-about of the effect-event. ${ }^{1}$

1 That in sufficient causation the cause determines the effect does not entail that the cause determines all by itself the effect. But it does entail that the cause determines the effect given the factual background (including laws of nature, circumstances, perhaps divine assistance, etc.). To determine means to guarantee: we have no instance of 
In what follows, the phrase 'a cause of' will always mean the same as 'a sufficient cause of', and 'to cause' will always mean the same as 'to be a sufficient cause of'. And these phrases will always be understood to exclude self-causation: Nothing is a cause of itself. (One may wish to count this as axiom (A0).)

The definition of the concept that is central to this paper is this:

(D) A first cause is a cause without a cause, in other, fully explicit words: a first sufficient cause is a sufficient cause (of some event), but a sufficient cause that itself has no sufficient cause.

It is easily seen:

(T1) If agents are not events, then every agent that is a cause is a first cause.

Suppose we have an agent that is a cause, i.e., that causes some event. If agents are not events, then that agent is not an event, hence it has no cause (for otherwise it would be an effect, and therefore an event, since effects are always events according to (A1)).

Now indeed:

(A2) Agents are not events, but substances. ${ }^{2}$ And therefore:

(T2) Every agent that is a cause is a first cause. Hence:

(T3) If there are agents that are causes, then there are first causes. But are there agents that are causes? That there are such items is doubted by many, even denied. Doubtless, however, there are events that are causes. And if one could find an event that is a cause, but has no cause, then this causal event - though not a causal agent - would also serve as a perfect first cause. But are there events that are causes without having a cause? We do not have purely scientific evidence for the existence of such events. What we do have purely scientific evidence for is merely this:

sufficient causation if the effect might still have failed to come about even though the cause (agent or event) spent its efficiency (assisted by the factual background); in that case, the cause just wasn't 'enough' for the effect.

${ }^{2}$ No substance is an event, no event a substance. Events have an intrinsic spatiotemporal location and normally also an intrinsic spatiotemporal extension (the exception with respect to the latter trait are momentary point-events). Substances do not have an intrinsic spatiotemporal location. Yet substances, too, are concrete individuals. Note that a substance is always capable of causal activity. Thus, all substances are potential agents. 
(A3) Some physical events are causes, but there is no physical event that causes them. ${ }^{3}$

Now, at this point, there is a crucial decision to be made in causationtheory. It is not an empirical, it is not a scientific, it is not a conceptual decision; it is a genuinely metaphysical decision. A choice is to be made between two very plausible metaphysical principles. One of these two principles is known as the principle of sufficient causation:

(A4.1) Every event has a cause.

The other principle is one of the principles known as principles of physical causal closure:

(A4.2) Every physical event that has a cause is caused by a physical event.

One cannot adopt both principles - because, unfortunately, their conjunction is incompatible with (A3). On the other hand, each of the two principles under consideration has so many credentials on its side that it seems rationally inappropriate to reject both. Let's see what would be the consequences if one accepted the one, or the other.

(A4.2) is the modernization of a materialistic, or physicalistic, principle that emerged as a metaphysical side-effect of the rise of modern physics. This original principle is the following:

$\left(A 4.2^{*}\right)$ Every physical event is caused by a physical event.

This latter principle was adopted by all who, inspired by impressive scientific progress, considered a purely immanent world-view - a worldview without transcendence - to be the only rational world-view. The insertion of 'that has a cause' after 'Every physical event' - which is of no detriment to the original metaphysical motivation - became necessary due to the developments in physics in the 20th century; these developments made modern physics entail the falsity of $\left(\mathrm{A} 4.2^{*}\right)$. (A4.2), however, is left quite untouched by them.

Now, obviously, the conjunction of (A3) and (A4.2) logically entails that there are physical events that are causes, but have no cause. Thus, if we add (A4.2) to our list of axiomatic principles (and not (A4.1)), then

${ }^{3}$ What does it mean that we have purely scientific evidence for (A3)? It means that as far as we can see by following the rules of scientific methodology, our best bet is that (A3) is true. It may, of course, be the case that we just don't see far enough. But, so far, there is no reason that might make a substantial reality out of this mere possibility. Note that the mere inventing of theories that exclude (A3) is not a refutation of (A3). 
the existence of first causes is established. There are, then, first causes in the form of physical events that are causes without having a cause.

In contrast, it is a straightforward logical consequence of (A4.1) that no event is a first cause. For if an event is a cause, (A4.1) requires that it also be caused, that is: have a cause. (A4.1) is a principle that throughout the roughly 2500 years of the history of philosophy was almost universally accepted by philosophers as an absolute requirement of rationality, comparable in this to a law of logic. And when (A4.2*) became prominent in the philosophical consciousness (roughly 300 years ago), it at first peacefully coexisted there with (A4.1); indeed, one could regard (A4.2*) as a mere specialization of (A4.1), as merely spelling out what it is that (A4.1) means for physical events. All of this changed in the 20th century with the establishment of quantum physics and empirical cosmology, and hence of the scientific fact that is stated by (A3). (A3) refutes $\left(\mathrm{A} 4.2^{*}\right)$, and it also refutes the conjunction of (A4.2) which is logically weaker than $\left(\mathrm{A} 4.2^{*}\right)$ - with (A4.1). But (A3) neither refutes (A4.2) taken by itself, nor does it refute (A4.1) taken by itself. If, tentatively, we add (A4.1) to our list of axiomatic principles (and not (A4.2)), continuing thereby a very long philosophical tradition in favour of (A4.1), we get an interesting result: There are physical events that have a cause, though they are not caused by any physical event. ${ }^{4}$

Given (A3), one cannot adopt (A4.1) and (A4.2) together, and it does not seem rationally right to reject both. A choice, therefore, has to be made between these two principles. There is no argument that would rationally force one to choose (A4.1) rather than (A4.2). But since (A4.1) involves much less of a metaphysical commitment than (A4.2); since, in other words, the rational appeal of (A4.1) is more general than that of (A4.2), and less dependent on the rationality of a specific metaphysical motivation, I herewith adopt (A4.1) as axiomatic, and as a consequence change its label from '(A4.1)' to simply '(A4)':

(A4) Every event has a cause.

And with both (A4) and (A3) as axioms, we now have as a theorem:

\footnotetext{
${ }^{4}$ Moreover, (A4.1) entails the following: Every physical event that has no physical cause still has a cause. This is the positive metaphysical solution to the so-called measurement problem (or rather, the gateway to such a solution). The negative metaphysical solution of the measurement problem is entailed by A4.2: Every physical event that has no physical cause has no cause at all. Whether there is also a non-metaphysical, scientific solution to the measurement problem, a solution within physics, remains to be seen. (By speaking of 'solutions' here, I mean of course possible solutions.)
} 
(T4) There are physical events that have a cause, though they are not caused by any physical event.

But, of course, with (A4) as an axiom, there is no chance that an event is a first cause; if there are first causes, then they must be something else than events. In fact, they must be agents, since the following is true:

(A5) Every cause is an agent or an event.

(A5) makes it possible to derive:

(T5) Every first cause is an agent.

Assume that $\mathrm{X}$ is a first cause, and assume also that $\mathrm{X}$ is an event. But then, according to (A4), $\mathrm{X}$ has a cause, and is, therefore, not a first cause - contrary to the first assumption. Therefore (holding on to that assumption): $\mathrm{X}$ is not an event, and therefore: $\mathrm{X}$ is an agent (because of (A5), and because $\mathrm{X}$ is, qua first cause, also a cause).

I am well aware that some philosophers have proposed facts, or even properties, as causes. But causes must be causally effective, and a property, taken by itself, is not causally effective; a property is only then causally effective - in an analogical way - if it is had, exemplified, instantiated by an object in such a way that the resulting fact is causally effective. But a fact, in its turn, is only then causally effective - in a derivative, secondary way - if it is replaceable in this role by a causal event. Causation by facts, in other words, is reducible to causation by events. - There is, therefore, no substantial reason to reject (A5). ${ }^{5}$

But could there not be a cause that is neither an event nor an agent (and also not reducible to either an event or an agent)? If this is a possibility, then it is, at least so far and for us, an empty possibility: we have no idea how to flesh it out, how to substantiate it. Moreover, the possibility that (A5) might be wrong (if it is a possibility) does not by itself endanger the

5 What about combined causes? - A group of events that is a cause can be fused into an event that is a cause. A group of agents that is a cause is itself an agent (namely, a group- agent) that is a cause. A cause $\mathrm{C}$ that consists of events and agents can be represented by a pair consisting of the event which is the fusion of the events that go into $\mathrm{C}$, and of the group-agent that is made up by the agents that go into $\mathrm{C}$. (If $\mathrm{C}$ consists just of one event and one individual agent, then the $\mathrm{C}$-representing pair is, of course, $\mathrm{C}$ itself.) If one member of the pair is not necessary for causation, then the real cause turns out to be either an event or an agent; if both members of the pair are necessary for causation, then either the event or the agent in the pair are given the role of (sufficient) cause, while the other member of the pair is counted among the items of the factual background (see footnote 1). - Therefore: the existence of combined causes does not, in the end, refute (A5). 
truth of (A5). As far as we know, (A5) is true. True, it is only true as far as we know. But what we know is what we have to go by.

(T4) gives rise to the following considerations: Suppose $\mathrm{E}^{*}$ is one of the physical events that - according to (T4) - have a cause, though there is no physical event that causes them. Thus:

(a) $\mathrm{E}^{\star}$ is a physical event.

(b) $\mathrm{E}^{\star}$ has a cause.

(c) There is no physical event that causes $\mathrm{E}^{*}$. Hence, by making use of (A5), we have:

(d) $\mathrm{E}^{\star}$ has a cause that is a nonphysical event or an agent. Assume now the following additional axiomatic principles:

(A6) Every event that is caused by an event is also caused by an event that is not caused by any event.

(A7) For all $x, y$ and $z$ : if $x$ causes $y$, and $y$ causes $z$, then $x$ causes $z$. (A7) expresses the transitivity of (sufficient) causation - one of the most uncontroversial principles in causation theory. (A6), in turn, is the limit principle for the causation by events. This, to some, may seem a very problematic principle; it actually is no such thing. Suppose (A6) is wrong, and $\mathrm{E}$ is an event that is caused by an event, but there is no event that causes $\mathrm{E}$ and is not caused by any event. It is easily seen (employing (A7)) that a consequence of this supposition is the following: all causal chains of events that end with $\mathrm{E}$ are infinite or incomplete.

Suppose $\mathrm{C}$ is a causal chain of events which ends with $\mathrm{E}$ and which is neither infinite nor incomplete. (Note that for a normal conception of a causal chain i.e., for the exclusion of its being a loop - the truth of (A0) is necessary.) Since $\mathrm{C}$ is not an infinite (but a finite) causal chain of events, there is a first event in $\mathrm{C}$, call it ' $\mathrm{E}_{1}$ '. Since $\mathrm{C}$ is a complete causal chain of events, there is no event that causes $E_{1}$. Given the transitivity of causation (i.e., the truth of (A7)) and given that $\mathrm{C}$ ends with $\mathrm{E}, \mathrm{E}_{1}$ causes $\mathrm{E}$. Thus there is an event (namely, $\mathrm{E}_{1}$ ) that causes $\mathrm{E}$ and is not caused by any event - contradicting the supposition which introduced $\mathrm{E}$ in the first place.

Is this consequence of negating (A6) - namely, the consequence that, for some event E, all E-ending causal chains of events are infinite or incomplete - more reasonable a priori than (A6)? I think it is not. Is this consequence more reasonable on empirical grounds than (A6)? Again, I think it is not, certainly not given today's physics. 
Using the two principles last introduced, we obtain from (d):

(e) $\mathrm{E}^{\star}$ is caused by an agent.

The first alternative in (d) leads to the result that $\mathrm{E}^{\star}$ is caused by an agent, just as does (trivially) the second alternative in (d). Suppose the first alternative in (d) is true: $\mathrm{E}^{\star}$ is caused by a nonphysical event. With (A6) we obtain: $\mathrm{E}^{\star}$ is caused by an event $\mathrm{E}^{\prime}$ that is not caused by any event. ${ }^{6}$ But according to (A4): $\mathrm{E}^{\prime}$ has a cause, G. Since $E^{\prime}$ is not caused by any event, $G$ must be an agent (according to (A5)). Since $G$ causes $E^{\prime}$ and $E^{\prime}$ causes $E^{*}$, it follows according to (A7): $G$ causes $E^{*}$. Therefore: $\mathrm{E}^{\star}$ is caused by an agent.

Consequently we get on the basis of (T2):

(f) There is an agent that is a first cause.

And this result - since, ultimately, it is a logical consequence purely of the axiomatic principles (A0) to (A7) - is a theorem: a statement logically proven on the basis of those axioms:

(T6) There is an agent that is a first cause.

This result chimes perfectly with the penultimate result of what has traditionally, since Kant, been called 'the Cosmological Argument for the Existence of God'. But although Thomas Aquinas nonchalantly concludes from the penultimate conclusion of the Cosmological Argument - that there is a first cause (which Thomas certainly thought to be an agent) - its ultimate conclusion: that there is $\mathrm{God}^{7}{ }^{7}$ it must be emphasized that this a very problematic last step. Nothing in Thomas Aquinas's argument, and nothing in the modernization of it here presented: nothing in (T6) and the axiomatic principles on which (T6) is based, justifies the conclusion that this agent which is a first cause is God or even $a$ god.

But, of course, the modernized Cosmological Argument I have presented here can be strengthened. In order to see just at what point it can be strengthened, consider first the compact presentation of the argument as it is now:

(A0) Nothing is a cause of itself.

(A1) Effects are always events.

${ }^{6}$ Note that $\mathrm{E}^{\prime}$ must be a nonphysical event. Otherwise, $\mathrm{E}^{\prime}$ would be a physical event that causes $\mathrm{E}^{*}$ - contradicting (c).

7 'Ergo est necesse ponere aliquam causam efficientem primam: quam omnes Deum nominant' ( $S$. Th. I, qu. 2, a. 3; see the conclusion of the secunda via). Perhaps Aquinas, instead of committing a blatant non sequitur, is merely being modest here. Note that, after the colon, he is not saying 'quae Deus est' or 'quam omnes convenienter Deum nominant'. 
(A2) Agents are not events, but substances.

(A3) Some physical events are causes, but there is no physical event that causes them.

(A4) Every event has a cause.

(A5) Every cause is an agent or an event.

(A6) Every event that is caused by an event is also caused by an event that is not caused by any event.

(A7) For all $x, y$ and $z$ : if $x$ causes $y$, and $y$ causes $z$, then $x$ causes $z$. [together logically entail among other things]

(T6) There is an agent that is a first cause.

Replace now (A3) by $\left(\mathrm{A}^{*}\right)$ (leaving the other axioms - or premises just as they are):

$\left(A 3^{*}\right)$ The Big Bang is a physical event that is a cause, but there is no physical event that causes it.

The specific principle $\left(\mathrm{A}^{*}\right)$ is just as true from the point of view of modern physics - on the basis of purely scientific evidence - as the unspecific (A3). ${ }^{8}$ With it and the rest of the axioms as premises, one can logically deduce:

$\left(T 6^{*}\right)$ There is an agent that is a first cause of the Big Bang.

From $\left(\mathrm{A}^{*}\right)$ and (A4) we get: $\mathrm{BB}$ is a physical event that has a cause, but there is no physical event that causes BB. Let A be a cause of BB. According to (A5), A is an agent or an event.

In case $\mathrm{A}$ is an agent, $\mathrm{A}$ is not an event (according to (A2)), and therefore $\mathrm{A}$ is not an effect (according to (A1)), i.e., A is not caused, in other words: A has no cause. But A causes BB. Thus: there is an agent (namely, A) that is a first cause of BB. In case $\mathrm{A}$ is an event, it follows on the basis of (A6) that $\mathrm{BB}$ is also caused by an event that is not caused by any event. Let $\mathrm{E}^{\prime}$ be such an event. It follows on the basis of (A4) that there is a cause of $\mathrm{E}^{\prime}$, and on the basis of (A5) it follows that that cause (any such cause) can only be an agent (it cannot be an event, since $\mathrm{E}^{\prime}$ is not caused by any event). Let $\mathrm{A}^{\prime}$ be such an agent. $\mathrm{A}^{\prime}$ causes $\mathrm{E}^{\prime}$, and $\mathrm{E}^{\prime}$ causes $\mathrm{BB}$, and therefore (according to (A7)): $\mathrm{A}^{\prime}$ causes $\mathrm{BB}$. Moreover, since $\mathrm{A}^{\prime}$ is an agent, it is not an event (see (A2)), and therefore not an effect (see (A1)), i.e., $A^{\prime}$ is not caused, in other words: $A^{\prime}$ has no cause. Thus we have again: there is an agent (namely, $\mathrm{A}^{\prime}$ ) that is a first cause of $\mathrm{BB}$.

\footnotetext{
${ }^{8}$ Footnote 3 applies to $\left(\mathrm{A} 3^{\star}\right)$ just as much as it applies to (A3).
} 
An agent that is a first cause of the Big Bang - that is: of the initial event of the Physical World - does seem to be godlike. By excluding the causation of the same event (any event) by several agents - which is a plausible theoretical step - we can even obtain that there is one and only one agent that is a first cause of the Big Bang. Moreover, also in line with traditional theism, the agent that causes the initiation of all of space-time-energymatter can hardly be denied to be nonphysical. However, nothing so far shows that this agent is different from, say, what Schopenhauer called 'the Will', different from a blind, irrational, and basically evil - but nevertheless transcendent - source of the Universe. That the First Cause (at least of the beginning) of the Universe is different from such a being is a matter of faith. But, note, it is also a matter of faith that God Himself is different from such a being. ${ }^{9}$

Rational theology is not the only field of metaphysics in which first causes prove useful - action theory is another, including the philosophy of human freedom of action. I submit that the following principle of minimal self-determination as a necessary condition of freedom is true:

(A8) I have free will only if it is true of at least one act (i.e., behaviour) of mine that no cause of it is wholly outside of me.

Note that (A8) spells out a necessary condition of my having free will, not a sufficient one. It is uncontroversial among incompatibilists that (A8) is true - and it can be used for testing the hypothesis of my having free will (and of course also for testing the hypothesis of your having free will: just apply (A8) in your own case): Should science show (or make it plausible to assume) that for every act (behaviour) of mine it is true that some cause of it is wholly outside of me, then I do not have free will. I do not know how compatibilists might be right in asserting that I could have (proper) free will even if for every act of mine it were true that a cause of it is wholly outside of me. (Remember that 'cause' always means sufficient cause here.)

${ }^{9}$ How does agent-causation stand to creation? Agent-causation is a logical species of creation qua making something actual. Every instance of agent-causation is ipso facto an instance of making something actual. The reverse is not true: if a substance is made actual, this is not an instance of agent-causation (since substances, being non-events, are never caused: see footnote 2 pertaining to (A2) and (A1)). Note that no instance of agentcausation is an instance of (creation qua) making something metaphysically possible, and that no instance of making something metaphysically possible is an instance of agentcausation. (But this may be trivially true: if there are no instances of making something metaphysically possible.) 
Suppose now that it is true of at least one act of mine that no cause of it is wholly outside of me. This does not mean that I have free will. But it does have interesting consequences. Let $\mathrm{D}^{\star}$ be an act of mine of which it is true that no cause of it is wholly outside of me. It is uncontroversial that there is an event that causes $\mathrm{D}^{*}$, and hence we can conclude on the basis of (A6) that there is an event $\mathrm{E}^{\star}$ that causes $\mathrm{D}^{\star}$ without being itself caused by any event. But according to (A4) $\mathrm{E}^{\star}$ has a cause, and since it cannot be an event that causes $\mathrm{E}^{\star}$, it must be an agent (according to (A5)). Let this agent be $A^{*}$. Since $A^{*}$ causes $E^{*}$, and $E^{*}$ causes $D^{*}$, it follows according to (A7): $A^{\star}$ causes $D^{\star}$. Now if $A^{\star}$ had a cause, $A^{\star}$ would be an effect, and therefore an event (see (A1)); but, following (A2), $A^{\star}$ is not an event (because it is an agent). Therefore: $A^{*}$ is not caused. And therefore: $\mathrm{A}^{\star}$ is an agent that is a first cause of $\mathrm{D}^{*}$. Since, according to supposition, no cause of $\mathrm{D}^{\star}$ is wholly outside of me, it follows moreover that $\mathrm{A}^{\star}$ is not wholly outside of me.

What has now been demonstrated is the following theorem:

(T7) If it is true of at least one act of mine that no cause of it is wholly outside of me, then it is also true of at least one act of mine that an agent that is not wholly outside of me is a first cause of that act.

And evidently the conjunction of (T7) and (A8) logically entails the following:

(T8) I have free will only if it is true of at least one act of mine that an agent that is not wholly outside of me is a first cause of that act.

Now, suppose I have free will and, as is required by (T8), there is at least one act of mine, $\mathrm{D}^{*}$, of which an agent, $\mathrm{A}^{*}$, that is not wholly outside of me is a first cause. Who might that agent be? Perhaps it is simply $I$ (I am, of course, not wholly outside of me). But, in fact, other possibilities seem to me more likely. In my view, a much more realistic position than the one I just considered is this: that I do not cause $\mathrm{D}^{\star}$, but that I am merely a part of $\mathrm{A}^{*}-\mathrm{a}$ necessary part, though: the rest of $\mathrm{A}^{*}$ does not by itself cause $\mathrm{D}^{\star}$. And who might the rest of $\mathrm{A}^{\star}$ consist of? Even if $\mathrm{A}^{\star}$ is an in itself minimal cause of $\mathrm{D}^{*}$, that is: a cause of $\mathrm{D}^{*}$ without a proper part of $\mathrm{A}^{*}$ being a cause of $\mathrm{D}^{\star}$, too - even then there still might be several monadic agents besides me in $\mathrm{A}^{\star}$. There seems to be no clue as to who they might be. But, I submit, God must be in $\mathrm{A}^{*}$ besides me; He, at least, is just as necessary to $\mathrm{A}^{\star}$ 's being a cause of $\mathrm{D}^{\star}$ as I am - I as chooser (choosing what is to be actualized: $\mathrm{D}^{*}$, thereby also choosing - implicitly - all the events that are causally instrumental for this, including, ultimately, 
a certain brain-event), He as enactor (implementing the actualization of whatever I chose to be actualized). ${ }^{10}$

Vis-à-vis these last ideas, which can only seem utterly speculative to most readers, I would like to emphasize that neither the axioms nor the theorems in this paper seem to me utterly speculative, epistemologically irresponsible, or irrational. I certainly believe that they provide food for serious thought. Thus, I propose that neither the existence of God nor the existence of human free will is a lost cause - because the existence of agents that are first causes is not a lost cause. ${ }^{11}$

Nevertheless, there are, of course, objections. I will consider three of them, two specifically against my modernization of the cosmological argument, one, more globally, against agent-causation.

Objection 1 (against $\left(\mathrm{A}^{*}\right)$ ): The Big Bang does not exist, because the Big Bang, if it is anything, is the total physical event which occurs at the first moment of time, and there is no first moment of time (as Stephen Hawking has famously held). Response: Even if there is no first moment of time, it does not follow that there is no initial physical event. Note that events, though they are required to be temporally finite according to the notion of event here employed (see the beginning of this paper), are not required by that notion to have a first or a last moment. An initial physical event is a physical event whose temporal region is the initial interval of time - and that interval may be an interval that is open on both sides. The Big Bang, then, is the total physical event whose temporal region is the initial interval of time. One might further object that there is not only no first moment of time, but also no initial interval of time. But, by the lights of modern physics (which may be wrong of course, but there is no guide known to be better), the initial interval of time is simply the first interval of time whose duration is the Planck-time (that is, $10^{-43} \mathrm{sec}$ ). There certainly is such an interval of time (even if there is no first moment of time), and the corresponding total event - the Big Bang - is, as far as we know, correctly described by $\left(\mathrm{A}^{*}{ }^{*}\right.$.

Objection 2 (against (A3) being the entire scientifically warranted truth): There is purely scientific evidence not only for (A3) but also for

${ }^{10}$ The result can be pictured as follows: $\{\mathrm{I}, \mathrm{God}\}=\mathrm{A}^{\star} \rightarrow \mathrm{E}_{1} \rightarrow \ldots \rightarrow \mathrm{E}_{\mathrm{N}} \rightarrow \mathrm{D}^{*}$.

${ }^{11}$ It is interesting to note that, in the presence of $(\mathrm{A} 0)$ and (A7), only by the introduction of agent-causation two principles can be maintained together that both seem central to explanatory rationality: the principle of sufficient causation and the first-cause principle: if there is a cause of an event, then there is also a first cause of it. (Both principles together were maintained by Thomas Aquinas, as were (A0) and, implicitly, (A7).) 
the existence of physical events that are causes without having a cause. For it is a scientific principle that if a physical event is not caused by any physical event then it is not caused by anything. Response: The objection relies on (A4.2) - a principle of physical causal closure - being a scientific principle. No doubt, many scientists employ it; but that, by itself, does not make it a scientific principle. ${ }^{12}$ In fact, (A4.2) is not a scientific, but a metaphysical principle - just like (A4.1), the principle of sufficient causation. It is a metaphysical principle because logical, mathematical, empirical, and esthetical considerations alone are not sufficient for warranting its assumption.

Objection 3: The notion of agent-causation, which is necessary for obtaining (T6), $\left(\mathrm{T}^{*}\right)$, and (T8), is an irremediably unclear notion. When, for example, does it happen? Or how is a purely agent-caused event different from a chance-event? Response: This is a stock objection, the merits of which are doubtful. I, for my part, have offered detailed analysis of agent-causation in my books Ereignis und Substanz and The Two Sides of Being, in the former regarding both creatural and divine agency, in the latter regarding only creatural agency. A comprehensive theory of causation, both of causation by events and causation by agents, can be found in my book Theorie der Kausalität, also containing extensive discussions of the literature. Some of the main results of Theorie der Kausalität are presented in my paper 'Causation in a New Old Key'. The emergence of creatural agent-causation in the course of natural history is defended in several of my papers, for example, 'The Emergence of Rational Souls' and 'New Perspectives for a Dualistic Conception of Mental Causation'. - And when does agent-causation 'happen'? Instances of agent-causation do not happen, since they - in contrast to the effects involved in them - are not events (and only events can happen). But if one absolutely wishes to assign a time to an instance of agent-causation, then it is simply the time of the effect that is involved in it.

And, finally, how is a purely agent-caused event ${ }^{13}$ different from a chance-event? This rhetorical question - its implicit assertion is: a purely

${ }^{12}$ Many scientists in the past have made successful use - within the very context of their scientific endeavours - of the hypothesis that God exists and has created the Universe (for example, Johannes Kepler in his arduous search for the laws of planetary motion). But that, of course, does not imply that the existence of God is a scientific principle.

${ }^{13}$ Consider the diagram in footnote 10: the purely agent-caused event in this diagram is $\mathrm{E}_{1}$ (the brain-event), not $\mathrm{D}^{*}$ (the behaviour). A purely agent-caused event is an event 
agent-caused event is in no way different from a chance-event - is meant to discredit the use of the notion of agent-causation in the incompatibilist analysis of free will, since - as is agreed on all sides - action on the basis of free will is not behaviour on the basis of mere chance. But the friend of agent-causation can react to the question in a wholly satisfactory way as follows. If a physical event is not caused by any physical event (and there are such events), then, in principle, it may have no (sufficient) cause at all; in that case (and only in that case) it is an event of causal chance: an event that happens without a sufficient cause (an event that 'just happens'). But since a purely agent-caused physical event does, after all, happen with a sufficient cause, it certainly is not an event of causal chance. We therefore have at least one way (illustrated for physical events) in which a purely agent-caused event is different from a chance-event.

However, the notion of chance-event can also be construed in a different way than as event of causal chance. The notion of chance-event can also be construed as event of teleological chance. If a physical event is not caused by any physical event, then, in principle, it may so turn out that there is no non-causal ground for it, in other words: that there is no reason for it; in that case (and only in that case) it is an event of teleological chance: an event that happens without a reason. But a purely agent-caused physical event is normally caused by its agent for a specific reason (one that is given in the consciousness or, more generally speaking, in the mind of that agent), ${ }^{14}$ and thus it is, normally, not an event of teleological chance, since there is a reason for it. ${ }^{15}$ I allow that sometimes a purely agent-caused physical event may be caused by the agent for no reason at all. In such a case we have an event of teleological chance before us (though not an event of causal chance). But this eventuality does not justify the sweeping unqualified judgment that a (i.e., any) purely agentcaused event is in no way different from a chance-event.

which is such that all of its causes are agents.

${ }^{14}$ Note that the reason for causing an event is often present in the mind of the agent without the caused event itself being present (as an intended event) in the mind of the agent. I lift my arm (that is: I cause my arm to rise) in order to make the chairman attend to me; this reason (i.e., non-causal ground) for my action is also the reason for my causing (with the help of God) the initial brain-event in the execution of that action; but that brain-event is certainly not present (as an intended event) in my mind.

${ }^{15}$ It is all-important to keep in mind that the event in question is not caused by that reason but is caused by the agent for that reason. Using Aristotelian terminology, one might say that the agent is the causa efficiens, not the causa finalis of the event, the reason the causa finalis, not the causa efficiens. 
Perhaps those that assert that a purely agent-caused event is in no way different from a chance-event mean to say something quite different from what they actually say. Perhaps they mean to say that a purely agentcaused event is in no way empirically distinguishable from a chance-event? But this, too, is untenable. A purely agent-caused physical event will not normally be an event of teleological chance (see above); and its not being an event of teleological chance can, at least sometimes, be made empirically apparent (an apparent purposeful context is found into which it fits). A physical chance-event, on the other hand, is always (at least) an event of teleological chance, because as a chance-event it is an event of causal chance or of teleological chance, and if it is an event of causal chance, then necessarily also an event of teleological chance. But also this character of being (at least) an event of teleological chance, necessarily belonging to any chance-event, can be made empirically apparent at least sometimes (no apparent purposeful context is found into which it would fit). Empirical appearances, it is true, only provide likelihoods, and always leave open the possibility that reality is really other than it appears to be. But if philosophers, who doubt that concept $\mathrm{X}$ is empirically distinguishable (in application) from concept $\mathrm{Y}$, require the empirical distinguishability of $\mathrm{X}$ from $\mathrm{Y}$ for the legitimacy of $\mathrm{X}$ as a concept, then they are ipso facto bound to accept this (the aforesaid) epistemological limitation.

Perhaps those that assert that a purely agent-caused event is in no way different from a chance-event mean to say something else yet: that a purely agent-caused event and an event of causal chance are intrinsically and in event-causal function in no way different from each other. There is truth in this, but it is almost trivial. The first event of a, in the direction of causes, finite and complete causal chain of events could, in principle, be an event of causal chance; event of causal chance is one kind of event without cause-event. However, the first event of a finite and complete causal chain of events could, in principle, also be a purely agent-caused event; purely agent-caused event is another kind of event without cause-event. There are no other kinds of event without cause-event. Suppose, then, event $\mathrm{E}^{\star}$ is the first event of a finite and complete causal chain of events. $E^{\star}$ is, to our best knowledge, an event without a cause-event. But whether that event is, in this function, a purely agent-caused event or, on the contrary, an event of causal chance, intrinsically there is no way of telling. Intrinsically and in event-causal function (i.e., qua event without cause-event), $\mathrm{E}^{\star}$ as a purely agent-caused event is in no way different from $\mathrm{E}^{\star}$ as an event of causal chance. 
APPENDIX: The principles (definitional, axiomatic, derived, or alternative) and logical relations considered in the paper

(A0) Nothing is a cause of itself.

(A1) Effects (i.e., what is caused) are always events.

(D) A first cause is a cause without a cause.

(T1) If agents are not events, then every agent that is a cause is a first cause.

(A2) Agents are not events, but substances.

(T2) Every agent that is a cause is a first cause.

(T3) If there are agents that are causes, then there are first causes.

(A3) Some physical events are causes, but there is no physical event that causes them.

(A4.1) Every event has a cause.

(A4.2) Every physical event that has a cause is caused by a physical event.

$\left(A 4.2^{*}\right)$ Every physical event is caused by a physical event.

(A4) Every event has a cause.

(T4) There are physical events that have a cause, though they are not caused by any physical event.

(A5) Every cause is an agent or an event.

(T5) Every first cause is an agent.

(a) $\mathrm{E}^{\star}$ is a physical event. [(T4)-based existential instantiation]

(b) $\mathrm{E}^{\star}$ has a cause. [ditto]

(c) There is no physical event that causes $\mathrm{E}^{\star}$. [ditto]

(d) $\mathrm{E}^{*}$ has a cause that is a nonphysical event or an agent. [(b), (c), (A5)]

(A6) Every event that is caused by an event is also caused by an event that is not caused by any event.

(A7) For all $x, y$ and $z$ : if $x$ causes $y$, and $y$ causes $z$, then $x$ causes $z$.

(e) $\mathrm{E}^{\star}$ is caused by an agent. [(d), (A6), (A4), (A5), (A7)]

(f) There is an agent that is a first cause. [(e), (T2)]

(T6) There is an agent that is a first cause.

(A0), (A1), (A2), (A3), (A4), (A5), (A6), (A7) logically implies (T6).

$\left(A 3^{*}\right)$ The Big Bang is a physical event that is a cause, but there is no physical event that causes it.

$\left(T 6^{*}\right)$ There is an agent that is a first cause of the Big Bang. 
(A0), (A1), (A2), (A3*), (A4), (A5), (A6), (A7) logically implies (T6*).

(A8) I have free will only if it is true of at least one act (i.e., behaviour) of mine that no cause of it is wholly outside of me.

(T7) If it is true of at least one act of mine that no cause of it is wholly outside of me, then it is also true of at least one act of mine that an agent that is not wholly outside of me is a first cause of that act.

(T8) I have free will only if it is true of at least one act of mine that an agent that is not wholly outside of me is a first cause of that act. ${ }^{16}$

\section{BIBLIOGRAPHY}

Meixner, U., Ereignis und Substanz. Die Metaphysik von Realität und Realisation (Paderborn: Schöningh, 1997)

Meixner, U., Theorie der Kausalität. Ein Leitfaden zum Kausalbegriff in zwei Teilen (Paderborn: Mentis, 2001)

Meixner, U., The Two Sides of Being. A Reassessment of Psycho-Physical Dualism (Paderborn: Mentis, 2004)

Meixner, U., 'Causation in a New Old Key', in Studia Logica, 76 (2004), 343-383 Meixner, U., 'New Perspectives for a Dualistic Conception of Mental Causation', in Journal of Consciousness Studies, 15, No. 1 (2008), 17-38

Meixner, U., 'The Emergence of Rational Souls', in Emergence in Science and Philosophy, edited by A. Corradini and T. O'Connor (New York/London: Routledge (Taylor \& Francis), 2010), pp. 163-179

${ }^{16}$ Acknowledgment: This paper was originally presented in Munich, at a conference for the Analytic Theology Project, generously funded by the John Templeton Foundation. 